\title{
A urgência da Saúde Coletiva na formação em Educação Física: lições com o COVID-19
}

\author{
The urgency of Public Health in Physical Education training: \\ lessons from COVID-19
}

Mathias Roberto Loch (https://orcid.org/0000-0002-2680-4686) ${ }^{1}$

Cassiano Ricardo Rech (https://orcid.org/0000-0002-9647-3448) ${ }^{2}$

Filipe Ferreira da Costa (https://orcid.org/0000-0003-3632-9310) ${ }^{3}$

${ }^{1}$ Programa de PósGraduação em Saúde Coletiva. Universidade Estadual de Londrina. Av. Robert Koch 60, Operária. 86038-350 Londrina PR Brasil.

mathiasuel@hotmail.com ${ }^{2}$ Departamento de Educação Física, Universidade Federal de Santa Catarina. Florianópolis SC Brasil.

${ }^{3}$ Grupo de Estudos e Pesquisas em Epidemiologia da Atividade Física. João Pessoa PB Brasil.

\begin{abstract}
The COVID-19 pandemic has generated several controversies in the health area, particularly regarding social isolation measures, widely perceived as being one of the most effective strategies to reduce the spread of the virus. The Physical Education (PE) area became involved in these discussions, through contradictory positions of professionals, scientific societies and class entities regarding the reopening of fitness centers during the pandemic. We understand that some of these discussions revealed important weaknesses in relation to the approach to basic health knowledge, such as those related to epidemiology and public health measures. We seek in this essay, without the intention of exhausting the subject or performing an academic prescription, to support our position regarding the urgency of the approach of PE training within the field of Public Health, as well as presenting some proposals for this approach to effectively occur. We advocate training that favors a broader view of health, that enables professionals in the field to understand the potential relationship between PE and health, but at the same time recognize that physical activity is not a panacea and that human health has many others determinants and conditions.

Key words Professional Training, Health Personnel, Physical Education and Training, Coronavirus Infections, Professional Practice
\end{abstract}

Resumo A pandemia de COVID-19 gerou diversas polêmicas na área da saúde, particularmente sobre as medidas de isolamento social, entendida como uma das estratégias mais eficazes para reduzir a propagação do vírus. A área da Educação Física (EF) se envolveu nessas discussões, por meio de posicionamentos contraditórios de profissionais, sociedades científicas e entidades de classe a respeito da reabertura das academias de ginástica em plena pandemia. Entendemos que alguns destes discursos revelaram importantes fragilidades com relação à aproximação aos conhecimentos básicos de saúde, como aqueles relativos à epidemiologia e medidas sanitárias. Buscamos neste ensaio, sem a intenção de esgotar o assunto ou realizar receituário acadêmico, sustentar nossa posição a respeito da urgência da aproximação da formação em EF com o campo da Saúde Coletiva, bem como apresentar algumas proposições para que ela, de fato, aconteça. Defendemos assim uma formação que favoreça uma visão mais ampliada da saúde, que possibilite que profissionais e professores compreendam a relação potencial entre a $E F$ e a saúde, mas que ao mesmo tempo reconheçam que a atividade física não é uma panaceia e que a saúde humana tem muitos outros determinantes e condicionantes.

Palavras-chave Capacitação Profissional, Pessoal de Saúde, Educação Física e Treinamento, Infecções por Coronavirus, Prática Profissional 


\section{Introdução}

A pandemia de COVID-19 surpreendeu o mundo e colocou luz sobre disputas ideológicas, geopolíticas, metodológicas e científicas no campo da saúde Global, que devem resultar em novas necessidades na formação e atuação profissional de saúde ${ }^{1}$.

Neste texto defendemos a urgência da discussão sobre maior participação do campo da Saúde Coletiva nos cursos de formação em Educação Física (EF). Entre os motivos que justificam essa "urgência" destacamos:

- A EF necessita para além de uma formação pautada nos efeitos clínicos da atividade física nas doenças, ampliar sua reflexão sobre elementos da gestão em saúde e determinantes socioambientais e políticos de saúde. Essa é uma demanda atual e apontada, entre outros documentos, pela Resolução do Conselho Nacional da Educação ${ }^{2,3}$ que reforça o campo da saúde como um eixo de formação para os novos currículos em formação em EF.

- A falta de clareza em muitos conceitos e argumentos utilizados por alguns gestores, dirigentes e profissionais da área de $\mathrm{EF}$, durante a Pandemia do COVID-19, sobre aspectos básicos em saúde, que, no nosso entendimento, deveriam estar incorporados ao fazer-profissional da área. Desse modo, fica evidente a necessidade de ir além do simples discurso de que EF faz parte da área da saúde.

Assim, sem a intenção de esgotar o assunto ou realizar receituário acadêmico, esse texto busca sustentar nossa posição a respeito da urgência da aproximação da formação em EF com o campo da Saúde Coletiva, bem como apresentar algumas proposições para que ela, de fato, aconteça. Entendemos que o cenário atual é propício para esta reflexão e que esta aproximação não será positiva somente para aqueles que pretendem atuar no Sistema Único de Saúde (SUS), mas para todos os professores e profissionais de EF, pois estes necessitam de uma visão mais ampliada de saúde em seus diferentes contextos de atuação profissional.

\section{Saúde Coletiva e Educação Física - Breves comentários sobre o cenário atual}

Antes de tecermos nossos comentários, esclarecemos que a EF não é a única área da saúde onde há uma aproximação frágil com a Saúde Coletiva. Não queremos aqui passar uma ideia de que a EF é o "vira-lata", que é a única área em que esta situação acontece, nem que a Saúde Coletiva seja um campo livre de críticas enquanto área do conhecimento. De fato, a Saúde Coletiva é "contra-hegemônica" na maioria dos cursos da área da saúde, uma vez que a formação ainda está fortemente atrelada ao modelo biomédico e baseada principalmente no atendimento das demandas específicas de cada área4.

Vale mencionar que a aproximação entre EF e Saúde Coletiva tem sido explorada em outros trabalhos ${ }^{3,5-8}$. Não é objetivo deste texto fazer uma síntese sobre esta questão, mas nos arriscamos a apontar que existem pontos que são relativamente consensuais a respeito desse tema: 1) nos últimos anos a EF, enquanto área do conhecimento, se aproximou da Saúde Coletiva, inclusive com o crescimento da epidemiologia da Atividade Física e com a formação de muitos profissionais em programas de pós graduação Stricto Sensu em Saúde Coletiva ou Epidemiologia e a aproximação de programas de pós-graduação da EF com temas próximos ao campo da Saúde Coletiva; 2) Em relação à EF enquanto profissão, a criação dos Núcleos de Apoio à Saúde da Família ${ }^{9}$ (agora intitulado Núcleo Ampliado de Saúde da Família e Atenção Básica - NASF-AB), representou um marco importante, pois materializou a possibilidade de inserção do Profissional de EF na Atenção Básica à Saúde e a realização do trabalho interprofisisonal neste contexto; 3) Relativo à formação profissional, aconteceram também avanços, mas estes ainda são pontuais, na maioria das vezes a partir da inserção de disciplinas nos currículos que abordam conteúdos como Epidemiologia e especificidades do SUS, o que, de fato, praticamente inexistia anteriormente.

Evidentemente que existem ameaças importantes a estes avanços. Nos últimos anos, uma série de ataques aconteceram ao SUS, alterando ou pelo menos flexibilizando o modelo de organização do sistema nos munícipios, modificando regras relativas ao financiamento entre outros ${ }^{10,11}$. Estas mudanças representam uma grande ameaça para um modelo de atenção que estava em processo de construção e também à inserção da EF, e de outras profissões, no SUS.

\section{A Educação Física e o COVID-19: desafios, simplismos e disputas}

Assim como buscamos deixar claro que a EF não é a única área com frágil aproximação com a Saúde Coletiva, destacamos também que não é difícil encontrar discursos frágeis sobre diversos pontos relacionados à temática do COVID-19, e 
outros temas de saúde, em profissionais de outras áreas da saúde.

Uma polêmica relacionada ao COVID-19 e que tem clara relação com a EF, foi a discussão sobre a reabertura ou não das academias de ginástica em plena pandemia. Os serviços de academias e clubes foram incluídos no rol de atividades essenciais em decreto presidencial ${ }^{12}$. Esta inclusão foi influenciada pelo pedido do Conselho Federal de $\mathrm{EF}^{13}$, e mesmo por algumas manifestações de conselhos regionais, de instituições privadas e profissionais da área em favor da reabertura de tais serviços (por exemplo: CREF/ RJ, CREF/PR; CREF/RS). Entendemos que muitos dos que defenderam a abertura das academias em plena pandemia, e em um cenário onde o Brasil apresentava um crescente número de casos e mortes pelo COVID-19, estavam muito preocupados com a manutenção dos seus empregos, muitas vezes precários, ou empresas. Por outro lado, os profissionais de EF não são os únicos afetados pela pandemia e pelo fechamento dos serviços/setores não essenciais, e consideramos que o enfrentamento deste problema, realmente delicado e complexo, deve se dar para além do nível individual e de cada setor da economia isoladamente. Aqui, nos alinhamos àqueles que entendem o Estado, em suas distintas esferas de governo, enquanto articulador e garantidor de estratégias e ações de curto, médio e longo prazo que deem suporte aos trabalhadores e empresas dos diferentes setores da economia, de modo a priorizar a manutenção dos empregos e a subsistência de sua população ${ }^{14}$.

Apesar de não termos o objetivo de sumarizar aspectos da "disputa" entre favoráveis e contrários à abertura das academias em plena pandemia, queremos usar alguns pontos para defender a questão central do texto, que é a urgência da Saúde Coletiva nos cursos de EF. Entre os argumentos apresentados pelos que defendiam a abertura das academias em plena pandemia estavam: a) a atividade física teria um efeito protetor para o COVID-19, pois sua prática melhoraria o sistema imunológico; b) Mais pessoas morrem no Brasil por problemas crônicos como hipertensão e diabetes, e que estas doenças estão relacionadas à inatividade física; c) $\mathrm{O}$ próprio $\mathrm{Mi}$ nistério da Saúde reconhece a importância da EF como profissão importante no enfrentamento da COVID-19, relacionando-a no rol de categorias que compõem a ação estratégica "O Brasil conta comigo"15.

Cada um destes pontos, admitidos por muitos como verdades absolutas, poderia ser con- frontada com outros argumentos, inclusive relacionados ao questionamento a respeito da relação entre intensidade das atividades e sistema imunológico, a "confusão" entre mortes por doenças crônicas com as mortes por COVID-19 e o fato de que, apesar do Ministério da Saúde ${ }^{15}$ abrir de fato a possibilidade de atuação de todos os profissionais da área da saúde, a atuação concreta na "linha de frente" é de profissionais de áreas como medicina e enfermagem, estes sim com papel central no tratamento e cuidados das pessoas com COVID-19.

Em alguns dos argumentos apresentados pelos defensores da abertura de academias, fica evidente o entendimento equivocado ou mesmo o não conhecimento de conceitos epidemiológicos básicos, como, por exemplo, causa suficiente, causa necessária, fator de risco e grupo de risco. Alguns parecem não compreender, por exemplo, que não fazer parte do grupo de risco não significa estar imune à uma determinada doença ou agravo à saúde, mas sim que a probabilidade disso acontecer é menor quando comparadas às pessoas que estão no grupo de risco. Assim, não há garantia que uma pessoa fisicamente ativa, caso venha a ter o COVID-19, não irá apresentar sintomas graves, mas sim que o risco de isso acontecer é menor, quando comparada ao grupo de risco.

A “disputa” entre os defensores e os contrários a abertura das academias em plena pandemia pode ser também visualizada nos comentários da postagem da Sociedade Brasileira de Atividade Física e Saúde, no facebook, no dia 14 de maio de $2020^{16}$, que se posicionou de maneira contrária à reabertura das academias. Tal postagem gerou comentários de apoio e compartilhamentos favoráveis, mas também críticas, algumas das quais com argumentos próximos aos já apresentados neste texto como, por exemplo, "morrem mais pessoas por causa da obesidade do que por causa do COVID-19".

Por fim, o último ponto abordado neste tópico diz respeito ao aconselhamento para a realização de atividades físicas em casa no período de pandemia. Não iremos nos ater demasiado ao tema, mas é necessário destacar que esta questão também foi tratada por muitos de maneira simplista. Entendemos que a realização destas práticas pode sim ser importante para a saúde, inclusive com foco na melhoria/manutenção da saúde mental, mas em muitas recomendações parecia haver mais um sentido de "ter que fazer", com caráter, portanto, "obrigatório”. Outro ponto ignorado em algumas destas "recomendações" diz respeito à aspectos como a habilidade das pessoas 
para a realização destas atividades, as preferências e gosto individual, as condições das residências, entre outros. Entendemos que estas recomendações, realizadas algumas vezes com um caráter persecutório e descontextualizadas da realidade de muitas pessoas é, em alguma medida, também reflexo da formação profissional em $\mathrm{EF}$, que não favorece um olhar mais ampliado de saúde.

\section{Saúde Coletiva nos cursos de formação em Educação Física - proposições para uma melhor aproximação}

Consideramos que esta parte do texto é a mais propositiva e, por isso, optamos por apresentar algumas proposições em tópicos, seguidos por breves comentários, que evidentemente não esgotam o tema.

- Não se faz uma aproximação, de fato efetiva, com o campo da Saúde Coletiva, somente com a criação e oferta de disciplinas mais específicas a este campo.

Apesar de representarem um avanço em relação ao que (não) existia anteriormente, um desafio importante é tornar a Saúde Coletiva algo mais estruturante na formação em EF e não algo que se aborda em momentos pontuais e com pouca aproximação com os demais conhecimentos.

Um possível caminho é fazer com que componentes curriculares que normalmente focam apenas em aspectos individualizados e centrados no mercado privado da atuação, busquem um olhar mais ampliado de saúde e dialoguem com a oferta e funcionamento dos serviços públicos de saúde. Além disso, outra questão fundamental é que os alunos sejam imersos na experiência, desde os primeiros semestres. Perspectivas conteudistas descoladas dos contextos do trabalho nos parecem antiquadas e insuficientes para a formação de um profissional autônomo, crítico, criativo e capaz de criar e transformar seus modos de trabalho.

Ademais, a formação deveria ir para além dos elementos técnicos científicos das áreas de conhecimento, e deve incluir aspectos que contribuiriam para que estudantes reconheçam o funcionamento dos principais Sistemas de Gestão das Políticas Públicas do país (ex: SUS, Sistema Nacional de Previdência Social, Sistema Nacional de Educação, entre outros). Somente a partir de uma compreensão ampliada da realidade, poderemos avançar em direção à uma formação mais humanizada, que contribuiria para se evitar discursos pouco conectados com as reais necessidades e condições de vida das pessoas.
- A aproximação com o campo da Saúde Coletiva não deve ser apenas para o curso de bacharelado em EF. O licenciado também precisa desta aproximação, pois o contexto escolar e a intervenção pedagógica demandam compreensões ampliadas de saúde.

É preciso superar a ideia, que nos parece comum na área, que tudo que se refere à saúde na EF diz respeito exclusivamente ao bacharel, enquanto os conteúdos relacionados à educação são importantes apenas para o licenciado.

Especificamente sobre a formação de professores de EF, acreditamos ser possível trabalhar questões relativas à, por exemplo, sexualidade, uso de substâncias, violência, mídias sociais, preconceitos de diferentes naturezas, além, é claro, de questões relacionadas ao cuidado de si e do outro, aos modos de viver e sua relação com a saúde individual e coletiva.

- A Saúde Coletiva se baseia no tripé: Epidemiologia, Ciências Humanas e Sociais, e Política e Planejamento.

Parece óbvio, mas ousamos afirmar que parte da EF interpreta Saúde Coletiva como sinônimo de epidemiologia, e considera que a aproximação da área com o campo da Saúde Coletiva se dá naturalmente e efetivamente pela simples abordagem de conteúdos da epidemiologia na formação. Não temos dúvida da importância da Epidemiologia na ampliação do olhar na formação em EF, e, inclusive, argumentamos anteriormente como a falta de uma melhor noção de conceitos básicos da epidemiologia resultou em discursos e posições que consideramos inadequadas e simplistas. Por outro lado, Ciências Humanas e Sociais e Política e Planejamento aparecem (quando aparecem) de maneira bem mais tímida na formação em EF e isso precisa ser modificado.

- O trabalho em saúde é interprofissional. Em geral a formação em EF, e em outras áreas, ainda se dá essencialmente na perspectiva uniprofissional e, por vezes, corporativista, valorizando demasiadamente saberes específicos de cada área, em detrimento da integração de saberes e das práticas interprofissionais colaborativas. $\mathrm{O}$ processo saúde-doença constitui fenômeno, por natureza, complexo, que demanda a mobilização de diferentes saberes e a organização coletiva das práticas de cuidado para atender às diferentes $\mathrm{e}$ complexas necessidades de saúde. O incentivo a composição de equipes multidisciplinares nos diferentes níveis de atenção à saúde não garante por si só a prática colaborativa no cuidado em saúde. Nesse sentido, entendemos que o aprendizado e desenvolvimento de práticas interpro- 
fissionais colaborativas desde a formação inicial são essenciais para a transformação dos modos de organização do trabalho nos diferentes cenários de atuação, fundamentalmente nos serviços públicos de saúde, mas não limitado a estes. Aprender juntos a trabalhar juntos pode não só qualificar as práticas de cuidado em saúde, mas também prevenir alguns dos desvios e impropriedades de comunicação destacados ao longo desse texto.

\section{Considerações finais}

Apesar de não ter sido objetivo deste texto fazer uma análise sistemática dos discursos relativos à reabertura das academias de ginástica em plena epidemia de COVID-19, nos pareceu evidente, em vários posicionamentos de profissionais de
EF e mesmo em alguns posicionamentos de entidades de classe, que se faz urgente a busca por uma formação que favoreça uma visão mais ampliada da saúde, que faça com que professores e profissionais de EF entendam a relação potencial entre a EF e a saúde, em suas diferentes dimensões, mas ao mesmo tempo reconheçam que a atividade física não é uma panaceia, uma pílula mágica, uma vez que a saúde é uma dimensão humana extremamente complexa e com muitos determinantes e condicionantes.

Entendemos que a pandemia ajudou a revelar uma urgência que já existia, mas que ficou ainda mais exposta por meio da postura de parte da área da EF em relação à algumas situações. Assim, cabe aos coletivos de docentes e discentes refletir e agir no sentido de qualificar a formação inicial e continuada na área, particularmente num cenário de tantas incertezas que nos é apresentado.

\section{Colaboradores}

MR Loch concebeu a ideia do texto, redigiu a versão inicial do manuscrito e contribuiu com a versão final do texto. CR Rech e FF Costa realizaram a análise crítica da versão inicial e contribuíram, de maneira significativa, com a versão final do texto. 


\section{Referências}

1. Ventura DFL, Ribeiro HG, Giulio GM, Jaime PC, Nunes J, Bógus CM, Antunes JLF, Waldman EA. Desafios da pandemia de COVID-19: por uma agenda brasileira de pesquisa em saúde global e sustentabilidade. $\mathrm{Cad}$ Saúde Pública 2020; 36(4):e00040620.

2. Conselho Nacional de Educação (CNE). Resolução $\mathrm{n}^{\circ}$ 6, de 18 de dezembro de 2018. Institui Diretrizes Curriculares Nacionais dos Cursos de graduação em Educação Física e dá outras providências. Diário Oficial da União; 2018.

3. Costa FF. Novas diretrizes curriculares para os cursos de graduação em Educação Física: oportunidades de aproximações com o SUS? Rev Bras Ativ Fís Saúde 2019; 24:e0067.

4. Carvalho YM, Ceccim RB. Formação e Educação em Saúde: aprendizados com a Saúde Coletiva. In: Campos GWS, Bonfim JRA, Minayo MCS, Akerman M, Drumond Jr. M, Carvalho YM, organizadores. Tratado de Saúde Coletiva. São Paulo: Hucitec; 2012. p. 137-170.

5. Bagrichevsky M, Estevão A, Palma A. La Educación Física y su inserción en el campo de la Salud Colectiva en Brasil: en búsqueda de un diálogo crítico sobre interfaces, pertenencias y sentidos. Physis 2008; 18(1):123-142.

6. Fraga AB, Carvalho YM, Gomes IM. Políticas de formação em educação física e saúde coletiva. Trab Educ Saúde 2012; 10(3):367-386.

7. Nogueira JAD, Bosi MLM. Saúde Coletiva e Educação Física: distanciamentos e interfaces. Cien Saude Colet 2017; 22(6):1913-1922.

8. Loch MR, Dias DF, Rech CR. Apontamentos para a atuação do Profissional de Educação Física na Atenção Básica à Saúde: um ensaio. Rev Bras Ativ Fís Saúde 2019; 24:e0069.

9. Brasil. Ministério da Saúde (MS). Portaria no 154, de 24 de janeiro de 2008. Cria os Núcleos de Apoio à Saúde da Família. Diário Oficial da União 2008; 24 jan.

10. Brasil. Portaria no 2.436 de 21 de setembro de 2017. Aprova a Política Nacional de Atenção Básica, estabelecendo a revisão de diretrizes para a organização da Atenção Básica, no âmbito do Sistema Único de Saúde (SUS). Diário Oficial da União 2017; 22 set.
11. Brasil. Ministério da Saúde (MS). Portaria no 2.979, de 12 de novembro de 2019. Institui o Programa Previne Brasil, que estabelece novo modelo de financiamento de custeio da Atenção Primária à Saúde no âmbito do Sistema Único de Saúde (SUS). Diário Oficial da União 2019; 13 nov.

12. Brasil. Presidência da República. Decreto no 10.344 , de 11 de maio de 2020. Altera o Decreto $n^{\circ} 10.282$, de 20 de março de 2020, que regulamenta a Lei no 13.979, de 6 de fevereiro de 2020, para definir os serviços públi$\cos e$ as atividades essenciais. Diário Oficial da União 2020; 11 mai.

13. Conselho Federal de Educação Física (CONFEF). Ofício no 370/2020. Rio de Janeiro: CONFEF; 2020.

14. Marconi N. A falsa dicotomia e o papel do Estado. Rev Conj Econ 2020; 74(4):34-35.

15. Brasil. Ministério da Saúde (MS). Portaria no 639, de 31 de março de 2020. Dispõe sobre a Ação Estratégica "O Brasil Conta Comigo - Profissionais da Saúde", voltada à capacitação e ao cadastramento de profissionais da área de saúde, para o enfrentamento à pandemia do coronavírus (COVID-19). Diário Oficial da União 2020; 2 abr.

16. Sociedade Brasileira de Atividade Física e Saúde. Nota da Sociedade Brasileira de Atividade Física e Saúde sobre a realização de atividades físicas e práticas corporais no contexto atual de pandemia da Covid-19 [Internet]. 2020 [acessado 2020 Mai 10]. Disponível em: http:// www.sbafs.org.br/noticia/109/nota-da-sociedade -brasileira-de-atividade-fisica-e-saude-sobre-a-realizacao-de-atividades-fisicas-e-praticas-corporais-nocontexto-atual-de-pandemia-da-covid-19

Artigo apresentado em 10/06/2020

Aprovado em 11/06/2020

Versão final apresentada em 13/06/2020 